\section{Control of Jupiter's radio emission and aurorae by the solar wind}

\author{
D. A. Gurnett ${ }^{\star}$, W. S. Kurth ${ }^{\star}$, G. B. Hospodarsky ${ }^{\star}$, A. M. Persoon ${ }^{\star}$, \\ P. Zarka $\dagger$, A. Lecacheux $\dagger$, S. J. Bolton $\ddagger$, M. D. Desch $\S$, W. M. Farrell $\S$, \\ M. L. Kaiser\$, H.-P. Ladreiter\|, H. O. Rucker\|, P. Galopeaug, P. Louarn\#, \\ D. T. Young ${ }^{\star 2}$, W. R. Pryor ${ }^{\star \star} \&$ M. K. Dougherty $\dagger \dagger$
}

* Department of Physics and Astronomy, University of Iowa, Iowa City, Iowa 52242, USA

$\dagger$ Space Research Department, Observatoire de Paris, 92195 Meudon, France $\ddagger$ Jet Propulsion Laboratory, 4800 Oak Grove Drive, Pasadena, California 91109, $U S A$

$\$$ NASA/Goddard Space Flight Center, Greenbelt, Maryland 20771, USA

II Space Research Institute, Austrian Academy of Science, A-8010 Graz, Austria 9 Centre d'étude des Environnements, Terrestre et Planétaires, Université de

Versailles Saint-Quentin-en-Yvelines (CETP/UVSQ), 10-12 Avenue de l'Europe, F-78140 Velizy, France

\# Centre d'Etude Spatiale des Rayonnements (CESR-CNRS), 9 Avenue du Colonel Roche, Centre National de la Recherche Scientifique, 31028 Toulouse, France

Department of Atmospheric, Oceanic and Space Science, University of Michigan, Ann Arbor, Michigan 48109, USA

** Laboratory for Atmospheric and Space Physics (LASP), University of Colorado, Boulder, Colorado 80303, USA

$\dagger \dagger$ Blackett Laboratory, Imperial College of Science and Technology, London SW7 2BZ, UK

Radio emissions from Jupiter provided the first evidence that this giant planet has a strong magnetic field ${ }^{1,2}$ and a large magnetosphere ${ }^{3}$. Jupiter also has polar aurorae ${ }^{4}$, which are similar in many respects to Earth's aurorae ${ }^{5}$. The radio emissions are believed to be generated along the high-latitude magnetic field lines by the same electrons that produce the aurorae, and both the radio emission in the hectometric frequency range and the aurorae vary considerably ${ }^{6,7}$. The origin of the variability, however, has been poorly understood. Here we report simultaneous observations using the Cassini and Galileo spacecraft of hectometric radio emissions and extreme ultraviolet auroral emissions from Jupiter. Our results show that both of these emissions are triggered by interplanetary shocks propagating outward from the Sun. When such a shock arrives at Jupiter, it seems to cause a major compression and reconfiguration of the magnetosphere, which produces strong electric fields and therefore electron acceleration along the auroral field lines, similar to the processes that occur during geomagnetic storms at the Earth.

The Cassini fly-by of Jupiter on 30 December 2000 provided an opportunity to carry out coordinated measurements between the Cassini spacecraft, which is on its way to Saturn, and the Galileo spacecraft, which has been orbiting Jupiter since 1995. Figure 1 shows a frequency-time spectrogram obtained from the radio and plasma wave science (RPWS) instrument ${ }^{8}$ on Cassini during the approach to Jupiter. Several different types of radio emissions can be seen in this spectrogram, but the most prominent are the distinct arc-like features from about 0.5 to $16 \mathrm{MHz}$. These arc-like features were first clearly seen during the Voyager $1 \mathrm{fly}$-by of Jupiter ${ }^{9}$, and are believed to be generated at the local electron cyclotron frequency via the cyclotron maser mechanism ${ }^{10}$, which produces radiation nearly perpendicular to the local magnetic field. Usually, two types of arcs can be identified, those associated with Jupiter's moon Io $^{11}$, labelled 'Io-controlled', and those not associated with Io. The Io-controlled arcs are strongest in the decametric frequency range, and are produced by a system of Alfven waves excited by Io $^{12,13}$. The nonIo-controlled emissions are independent of Io's position and are highly variable. These emissions are often called hectometric radiation, because they mainly occur at hectometric wavelengths
(0.3-3 MHz), although some radiation also extends upward into the decametric frequency range $(3-30 \mathrm{MHz})$. Previous studies have shown that the hectometric radiation is generated at altitudes of several jovian radii along the high-latitude auroral field lines ${ }^{14}$.

In this study we have concentrated on investigating the solar wind control of jovian hectometric radiation and the auroral extreme ultraviolet emissions. Evidence already exists that the solar wind plays a role in controlling the intensity of the hectometric radiation ${ }^{15,16}$. To provide a parameter that characterizes the intensity of the jovian hectometric radiation, we have integrated the radio emission spectrums detected by the Cassini RPWS instrument and the Galileo plasma wave science (PWS) ${ }^{17}$ instrument over a frequency range from 0.5 to $5.6 \mathrm{MHz}$. The corresponding radiated power fluxes, in $\mathrm{W} \mathrm{m}^{-2}$, are shown in Fig. 2: large variations are present in the hectometric radio emission intensity. Three well defined periods of enhanced hectometric radiation are evident. These events are indicated by the shading, and are labelled events A, $\mathrm{B}$ and $\mathrm{C}$. The magnetometer and plasma measurements on Cassini show that each of these events is preceded by an interplanetary shock. The arrival times of the shocks at Cassini are indicated by the arrows at the top of the plot.

Further details of event A are shown on an expanded timescale in Fig. 3. The arrival of the interplanetary shock at Cassini is clearly indicated by the jump in the magnetic field at the arrow marked 'shock' in Fig. 3a. The shock is followed by a region of strongly enhanced magnetic field lasting about 5 days, with a peak on day 326. Figure $3 \mathrm{~b}$ shows that the ion density increased markedly over the same period, indicating that the shock is followed by a region of highly compressed plasma. Such regions of compressed plasma are typical of interplanetary shocks at this distance from the Sun. The same interplanetary shock was subsequently detected by the Galileo plasma wave instrument near Jupiter about $16 \mathrm{~h}$ later at 09:15 UT on day 324 . From the approximately 16-h travel time from Cassini (at $x=+540 R_{\mathrm{J}}$, where $x$ is the distance toward the Sun from Jupiter and $R_{\mathrm{J}}$ is the radius of Jupiter) to Galileo (at $x=-52 R_{\mathrm{J}}$ ), the average shock propagation speed is estimated to be $721 \mathrm{~km} \mathrm{~s}^{-1}$. This speed is typical of a relatively strong interplanetary shock.

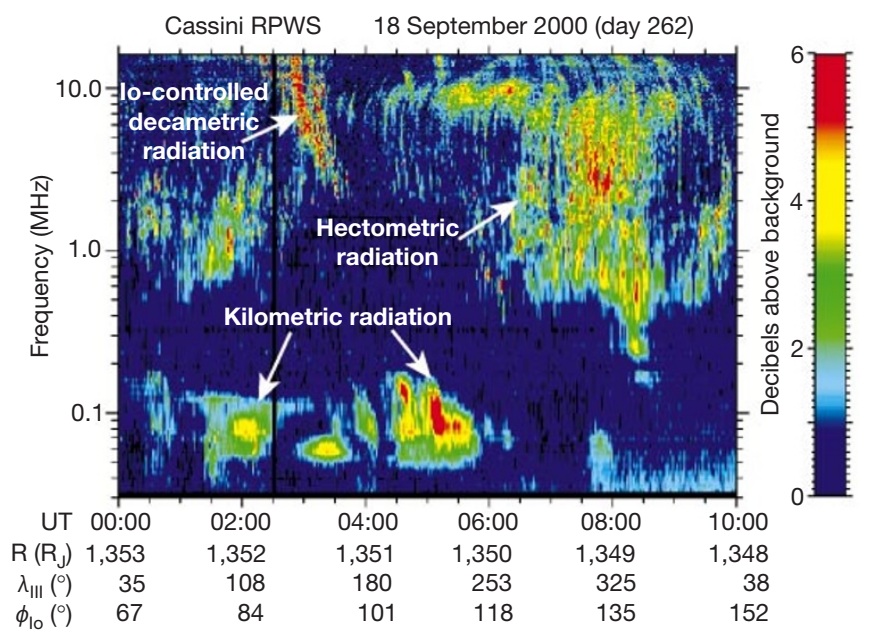

Figure $1 \mathrm{~A}$ representative frequency-time spectrogram of jovian radio emissions detected by the Cassini spacecraft during the approach to Jupiter at a radial distance of about 1,350 Jupiter radii $\left(R_{\mathrm{J}}\right)$. The colour bar on the right gives the radio emission intensities in decibels above the background noise level, which is approximately the cosmic background. UT, universal time at the spacecraft, in hours and minutes. $R$, the radial distance of the spacecraft from Jupiter in $R_{\mathrm{J}}$. $\lambda_{\| 1}$, the system III longitude of the spacecraft. $\varphi_{\mathrm{l}}$, the phase of lo relative to the spacecraft. Three types of jovian radio emissions are evident in this spectrogram: lo-controlled decametric radiation, hectometric radiation and kilometric radiation. For a review of these types of radio emissions and the associated terminology see ref. 6 . RPWS, radio and plasma wave science instrument. 


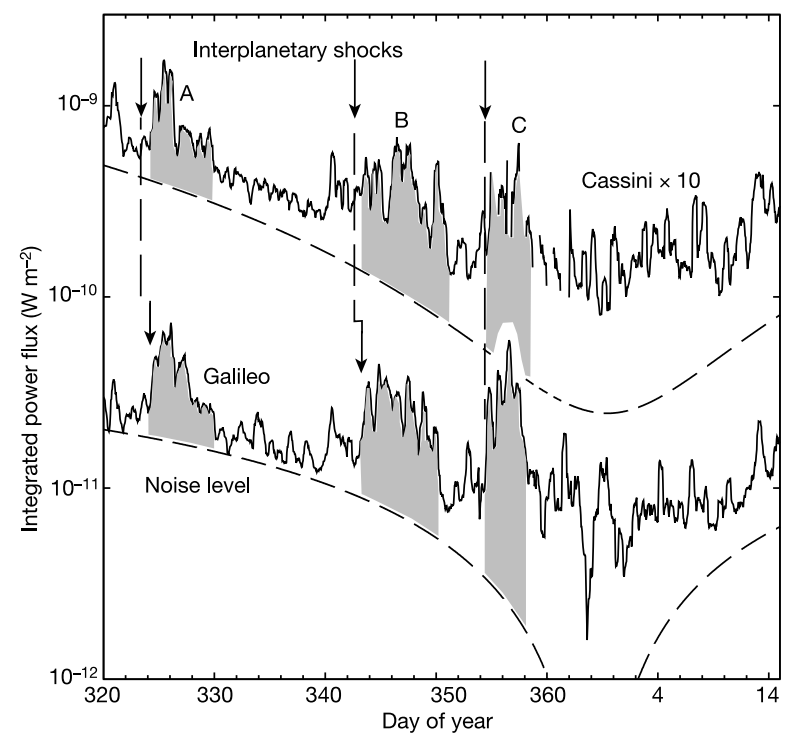

Figure 2 The power flux of the hectometric radiation as a function of time as detected by the Cassini and Galileo spacecraft during the Cassini fly-by of Jupiter. The power flux is computed by integrating the received spectrum from 0.5 to $5.6 \mathrm{MHz}$, averaging over the $9 \mathrm{~h} 55 \mathrm{~min}$ rotational period of Jupiter, and correcting to a radial distance of $100 R_{\mathrm{J}}$ using a $1 / R^{2}$ law. The Cassini plot has been shifted upward by a factor of ten $(\times 10)$ relative to the Galileo plot. Three strong hectometric radio emission events (labelled A, B and C) were detected during the Cassini approach to Jupiter. The first starts about midday on day 324 and extends to about day 330 , the second starts about day 343 and extends to about day 351 , and the third starts late on day 354 and extends to about day 358 Comparisons with the solar wind magnetic field and plasma data from the Cassini magnetometer (MAG) $)^{27}$ and plasma spectrometer (CAPS) ${ }^{28}$ show that event A was preceded by a strong interplanetary shock at $17: 92$ ur on day 323 , event $B$ was preceded by a strong interplanetary shock at 23:12 ut on day 342 , and event $C$ was preceded by a strong interplanetary shock at 15:12 ut on day 354 . The shocks associated with events A and $B$ were also subsequently detected by the Galileo plasma wave science (PWS) instrument at 09:15 ut on day 324 , and 12:10 ut on day 343. The shock associated with event $C$ could not be detected by Galileo, since the spacecraft was well inside the magnetosphere at the time this shock was expected to arrive at Jupiter.

Figure $3 \mathrm{c}$ shows that the arrival of the shock at Galileo (indicated by the arrow) is essentially coincident with the onset of the hectometric radio emission event. However, we note that it also takes nearly two days, to early in day 326 , before the hectometric radiation reaches peak intensity. When corrected for the solar wind travel time, the peak in the hectometric radiation intensity occurs at about the time of maximum solar wind density. Figure $3 \mathrm{~d}$ shows that the peak in the hectometric radio emission coincides almost exactly with a peak in the auroral extreme ultraviolet intensity, as detected by the ultraviolet spectrograph on Cassini.

An expanded-timescale frequency-time spectrogram of the radio emission intensities detected by the Galileo spacecraft during event $\mathrm{B}$ is shown in Fig. 4. During this period Galileo was very close to the magnetopause on the dusk side of the magnetosphere. The location of the magnetopause relative to the spacecraft can be determined from the presence or absence of trapped continuum radiation, which occurs at frequencies from about 0.5 to $10 \mathrm{kHz}$. Trapped continuum radiation is present when the spacecraft is inside the magnetosphere, and absent when the spacecraft is outside the magnetosphere ${ }^{18}$. An arrow indicating the time that the interplanetary shock associated with this event was detected by Cassini is shown at the top of the plot. At this time Galileo was clearly within the magnetosphere, as evidenced by the presence of the trapped continuum radiation. At 12:10 uT on day 343, approximately $13 \mathrm{~h}$ after the shock was detected by Cassini, the magnetopause abruptly moved inward across Galileo, as indicated by the

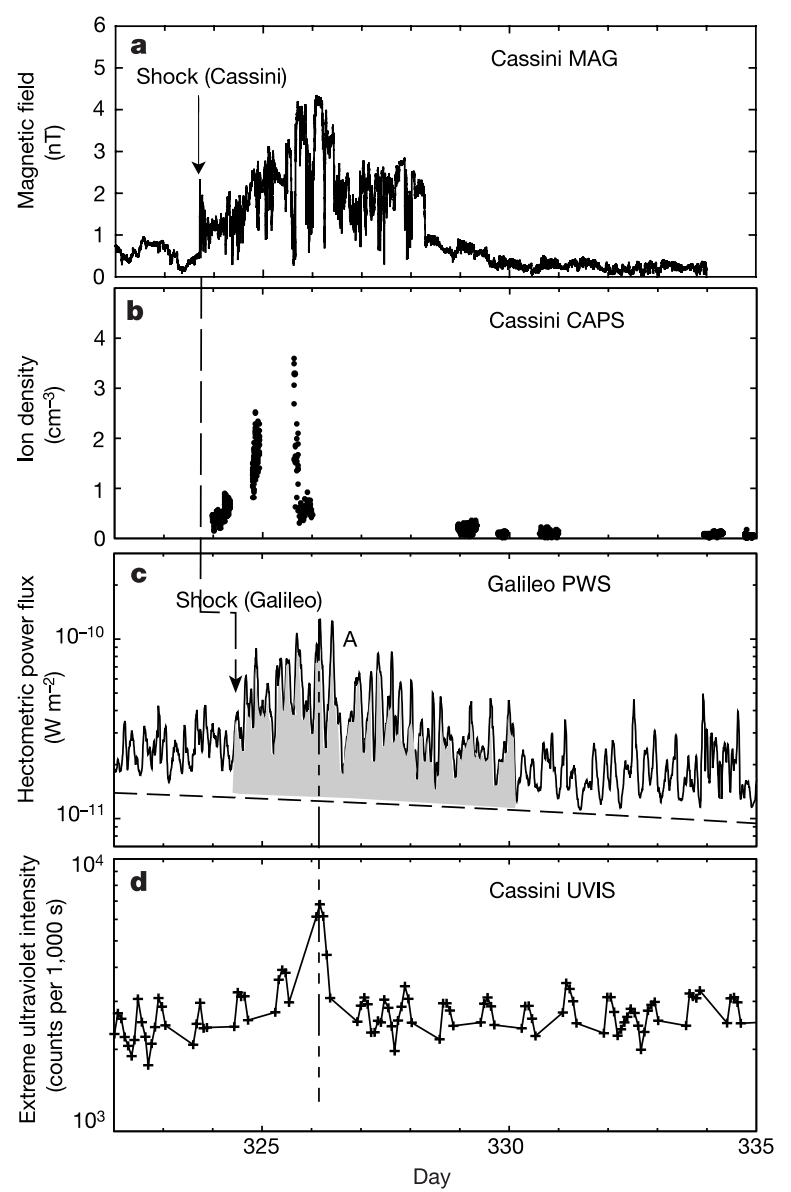

Figure $3 \mathrm{~A}$ detailed comparison of the solar wind magnetic field strengths. Data is from the Cassini MAG instrument (a), the solar wind ion densities from the Cassini CAPS instrument (b), the integrated $(0.5-5.6 \mathrm{MHz})$ hectometric radiation intensities (1-h averages) from the Galileo PWS instrument (c), and the disk-integrated extreme ultraviolet auroral $\mathrm{H} 2$ band (110.8-113.1 nm) intensities for the Cassini ultraviolet imaging spectrograph (UVIS) instrument ${ }^{29}$ (d) for event A. The arrows show the times at which the interplanetary shock was detected by Cassini and Galileo. We note that the onset of the hectometric radiation coincides almost exactly with the arrival of the shock at Galileo. Also the peak in the hectometric radiation intensity occurs at the same time as the maximum brightness of the extreme ultraviolet auroral emission, and near the time of maximum solar wind plasma density (after correcting for the approximately 13-h solar wind travel time).

sudden disappearance of the continuum radiation. Although the shock was not detected directly, this sudden compression was almost certainly caused by the arrival of the interplanetary shock. From the time difference between the arrival of the shock at Cassini (at $x=+324 R_{\mathrm{J}}$ ) and the onset of the compression at Galileo (at $x=-49 R_{\mathrm{J}}$ ) the average propagation speed is estimated to be $528 \mathrm{~km} \mathrm{~s}^{-1}$, which is typical of a low-Mach-number interplanetary shock. As indicated at the top of the spectrogram the onset of the magnetospheric compression corresponds almost exactly with the onset of hectometric radio emission event B.

The observations presented above provide strong evidence that jovian hectometric radiation and auroral extreme ultraviolet emissions are triggered by interplanetary shocks. The processes involved appear to be very similar to those occurring in Earth's magnetosphere. The comparable type of radio emission at Earth is auroral kilometric radiation, which is known to be closely associated with the terrestrial aurora ${ }^{19}$. Just as with the jovian hectometric radiation, the terrestrial kilometric radiation is highly variable and is closely correlated with magnetic storms ${ }^{20}$, including those triggered by interplanetary shocks. Both types of radio emissions are believed to be generated along the auroral magnetic field lines at comparable 

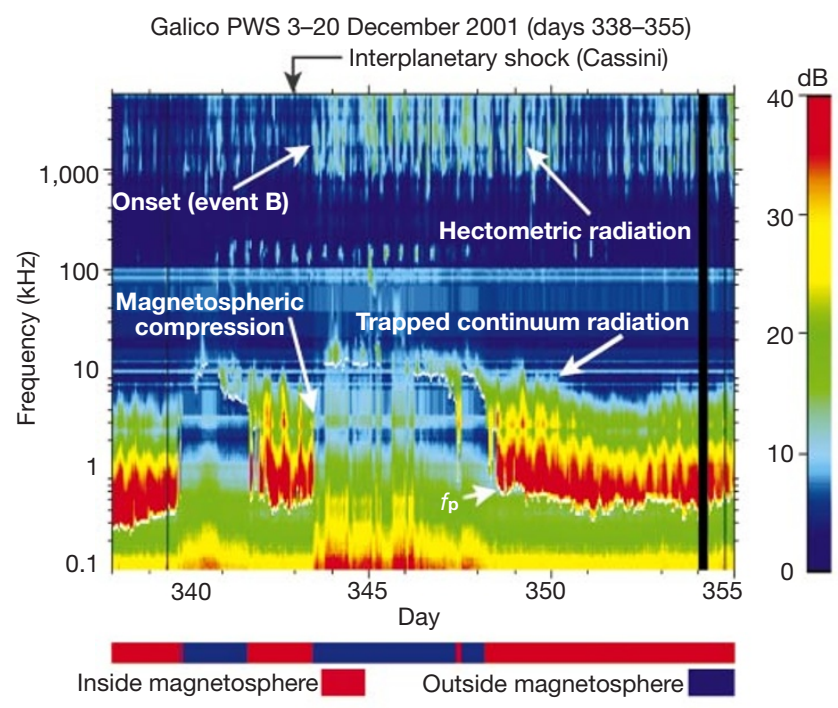

Figure 4 A frequency-time spectrogram from the Galileo PWS showing the radio emission intensities detected by Galileo during event B. The time of the interplanetary shock responsible for this event, as detected by Cassini (at 23:12 ut on day 342), is indicated by the arrow at the top of the spectrogram. The arrival of this shock at Jupiter is believed to be indicated by the disappearance of the trapped continuum radiation at 12:10 ut on day 343 , which indicates a sudden compression of the magnetosphere. The white line at the bottom edge of the trapped continuum radiation is the electron plasma frequency, which is proportional to the square root of the electron density. The colour bar at the bottom of the spectrogram indicates when the spacecraft is inside or outside the magnetosphere, on the basis of the presence or absence of the continuum radiation. As can be seen, the onset of the hectometric radiation occurs essentially coincident with the sudden magnetospheric compression on day 343.

altitudes ( 1 to 3 planetary radii) ${ }^{14,21}$ by the cyclotron maser mechanism ${ }^{10,22}$. No in situ measurements are available in the highlatitude auroral regions of the jovian magnetosphere, so we use our knowledge of terrestrial auroral processes to help us to understand the processes that are occurring in the jovian magnetosphere. For example, when an interplanetary shock interacts with Earth's magnetosphere, it is known that the magnetosphere becomes strongly compressed. The resulting compression leads to a largescale reconfiguration of the magnetic field and associated acceleration and energization of plasma within the magnetosphere. The stresses associated with the compression lead to large field-aligned currents and electric fields, particularly along the high-latitude magnetic field lines, where the electrons that carry the field-aligned currents strike the atmosphere and produce the aurora. Very detailed measurements now exist from Earth-orbiting satellites that show how the electrons involved in this process produce the terrestrial auroral kilometric radiation via the cyclotron maser mechanism ${ }^{23,24}$. We infer that similar processes are responsible for the jovian hectometric radiation. At Earth the auroral kilometric radiation is known to be generated along magnetic field lines that terminate in bright spots in the aurora ${ }^{25}$. Because the jovian aurora is also known to have considerable fine structure ${ }^{26}$, we can also infer that the discrete arc-like structure evident in the hectometric radiation (see Fig. 1) is most probably associated with the fine structure in the jovian aurora.

Received 3 August; accepted 1 November 2001.

1. Burke, B. F. \& Franklin, K. L. Observations of a variable radio source associated with the planet Jupiter. J. Geophys. Res. 60, 213-217 (1955).

2. Drake, F. D. \& Hvatum, S. Non-thermal microwave radiation from Jupiter. Astron. J. 64, 329-330 (1959).

3. Warwick, J. W. Radiophysics of Jupiter. Space Sci. Rev. 6, 841-891 (1967).

4. Broadfoot, A. L. et al. Extreme ultraviolet observations from Voyager 1 encounter with Jupiter. Science 204, 979-982 (1979)

5. Chamberlain, J. W. Physics of the Aurora and Airglow (Academic, New York, 1961).
6. Carr, T. D., Desch, M. D. \& Alexander, J. K. in Physics of the Jovian Magnetosphere (ed. Dessler, A. J.) 226-284 (Cambridge Univ. Press, Cambridge, 1983).

7. Clarke, J. T., Moos, H. W., Atreya, S. K. \& Lane, A. L. Observations from Earth orbit and variability of the polar aurora on Jupiter. Astrophys. J. 241, L179-L1182 (1980).

8. Gurnett, D. A. et al. The Cassini radio and plasma wave investigation. Space Sci. Rev. (in the press).

9. Warwick, J. W. et al. Voyager 1 planetary radio astronomy observations near Jupiter. Science 204, 995 998 (1979).

10. Wu, C. S. \& Lee, L. C. A theory of terrestrial kilometric radiation. Astrophys. J. 213, 621-626 (1979). 11. Bigg, E. K. Influence of the satellite Io on Jupiter's decametric radiation. Nature 203, 1008-1010 (1964),

12. Goldreich, P. \& Lynden-Bell, D. Io, a unipolar inductor. Astrophys. J. 156, 59-78 (1969).

13. Queinnec, J. \& Zarka, P. Io-controlled decametric arcs and Io-Jupiter interaction. J. Geophys. Res. 103, 26649-26666 (1998).

14. Ladreiter, H. P., Zarka, P. \& Lecacheux, A. Direction finding study of Jovian hectometric and broadband kilometric radio emissions: Evidence for their auroral origin. Planet. Space Sci. 42, 919931 (1994).

15. Zarka, P. \& Genoa, F. Low-frequency Jovian emission and solar wind magnetic sector structure. Nature 306, 767-768 (1983).

16. Desch, M. D. \& Barrow, C. H. Direct evidence for solar wind control of Jupiter's hectometerwavelength radio emission. J. Geophys. Res. 89, 6819-6823 (1984).

17. Gurnett, D. A. et al. The Galileo plasma wave investigation. Space Sci. Rev. 60, 341-355 (1992).

18. Scarf, F. L., Gurnett, D. A. \& Kurth, W. S. Jupiter plasma wave observations: an initial Voyager 1 overview. Science 204, 991-995 (1979).

19. Gurnett, D. A. The Earth as a radio source: terrestrial kilometric radiation. J. Geophys. Res. 79, 42274238 (1974).

20. Voots, G. R., Gurnett, D. A. \& Akasofu, S.-Y. Auroral kilometric radiation as an indicator of auroral magnetic disturbances. J. Geophys. Res. 82, 2259-2266 (1977).

21. Gallagher, D. L. \& Gurnett, D. A. Auroral kilometric radiation: time-averaged source location. J. Geophys. Res. 84, 6501-6509 (1979).

22. Zarka, P. Auroral radio emissions at the outer planets: observations and theories. J. Geophys. Res. 103, 20159-20194 (1998)

23. Louarn, P. et al. Trapped electrons as a free energy source for the auroral kilometric radiation. J. Geophys. Res. 95, 5983-5995 (1990).

24. Ergun, R. E. et al. Electron-cyclotron maser driven by charge particle acceleration from magnetic fieldalligned electric fields. Astrophys. J. 538, 456-466 (2000).

25. Huff, R. L. Mapping of auroral kilometric radiation sources to the aurora. J. Geophys. Res. 93, 1144511454 (1988).

26. Clarke, J. T. et al. Hubble Space Telescope imaging of Jupiter's UV aurora during the Galileo orbiter mission. J. Geophys. Res. 103, 20217-20236 (1998).

27. Dougherty, M. K. et al. The Cassini magnetic field investigation. Space Sci. Rev. (submitted).

28. Young, D. T. et al. Cassini plasma spectrometer investigation. Space Sci. Rev. (submitted).

29. Esposito, L. W. et al. The Cassini ultraviolet imaging spectrograph investigation. Space Sci. Rev. (in the press).

\section{Acknowledgements}

The research at the University of Iowa, the University of Michigan, and the University of Colorado was supported by NASA through the Jet Propulsion Laboratory.

Correspondence and requests for materials should be addressed to D.A.G. (e-mail: donald-gurnett@iowa.edu).

\section{Ultra-relativistic electrons in Jupiter's radiation belts}

S. J. Bolton ${ }^{\star}$, M. Janssen ${ }^{\star}$, R. Thorne $\dagger$, S. Levin ${ }^{\star}$, M. Klein ${ }^{\star}$, S. Gulkis, T. Bastian $\neq$, R. Sault $₫$, C. Elachi*, M. Hofstadter ${ }^{\star}$, A. Bunker ${ }^{\star}$, G. Dulk\|, E. Gudim ${ }^{\star}$, G. Hamilton ${ }^{\star}$, W. T. K. Johnson ${ }^{\star}$, Y. Leblanc\|, O. Liepack ${ }^{\star}$, R. McLeodg, J. Rollerg, L. Roth \& R. West $^{\star}$

* Jet Propulsion Laboratory/Caltech, Pasadena, California 91109, USA

$\dagger$ University of California, Department of Atmospheric Sciences, Los Angeles, California 90024, USA

$¥$ National Radio Astronomy Observatory, Charlottesville, Virginia 24944, USA $\$$ Australia Telescope National Facility, Epping, New South Wales 1710, Australia II Observatory of Paris, Department of Space Research, F-92195 Meudon, France Iewis Center for Educational Research, Apple Valley, California 92307, USA

Ground-based observations have shown that Jupiter is a twocomponent source of microwave radio emission ${ }^{1}$ : thermal atmospheric emission and synchrotron emission ${ }^{2}$ from energetic electrons spiralling in Jupiter's magnetic field. Later in situ measurements ${ }^{3,4}$ confirmed the existence of Jupiter's highenergy electron-radiation belts, with evidence for electrons at 